\title{
Lutetium-177-PSMA-I\&T as metastases directed therapy in oligometastatic hormone sensitive prostate cancer, a randomized controlled trial
}

Bastiaan M. Privé ${ }^{1}$, Marcel J. R. Janssen ${ }^{1}$, Inge M. van Oort ${ }^{2}$, Constantijn H. J. Muselaers², Marianne A. Jonker ${ }^{3}$, Michel de Groot ${ }^{1}$, Niven Mehra ${ }^{4}$, J. Fred Verzijlbergen', Tom W. J. Scheenen', Patrik Zámecnik', Jelle O. Barentsz', Martin Gotthardt ${ }^{1}$, Walter Noordzij ${ }^{5}$, Wouter V. Vogel ${ }^{6,7}$, Andries M. Bergman $^{8}$, Henk G. van der Poel ${ }^{9}$, André N. Vis ${ }^{10}$, Daniela E. Oprea-Lager ${ }^{11}$, Winald R. Gerritsen ${ }^{4}$, J. Alfred Witjes ${ }^{2}$ and James Nagarajah ${ }^{1 *}$ (D)

\begin{abstract}
Background: In recent years, there is increasing evidence showing a beneficial outcome (e.g. progression free survival; PFS) after metastases-directed therapy (MDT) with external beam radiotherapy (EBRT) or targeted surgery for oligometastatic hormone sensitive prostate cancer (OHSPC). However, many patients do not qualify for these treatments due to prior interventions or tumor location. Such oligometastatic patients could benefit from radioligand therapy (RLT) with ${ }^{177} \mathrm{Lu}$-PSMA; a novel tumor targeting therapy for end-stage metastatic castration-resistant prostate cancer (mCRPC). Especially because RLT could be more effective in low volume disease, such as the oligometastatic status, due to high uptake of radioligands in smaller lesions. To test the hypothesis that ${ }^{177} \mathrm{Lu}$-PSMA is an effective treatment in oHSPC to prolong PFS and postpone the need for androgen deprivation therapy (ADT), we initiated a multicenter randomized clinical trial. This is globally, the first prospective study using ${ }^{177}$ Lu-PSMA-I\&T in a randomized multicenter setting.
\end{abstract}

Methods \& design: This study compares ${ }^{177}$ Lu-PSMA-I\&T MDT to the current standard of care (SOC); deferred ADT. Fiftyeight patients with oHSPC ( $\leq 5$ metastases on PSMA PET) and high PSMA uptake (SUVmax > 15, partial volume corrected) on ${ }^{18}$ F-PSMA PET after prior surgery and/or EBRT and a PSA doubling time of $<6$ months, will be randomized in a 1:1 ratio. The patients randomized to the interventional arm will be eligible for two cycles of 7.4GBq ${ }^{177}$ Lu-PSMA-1\&T at a 6week interval. After both cycles, patients are monitored every 3 weeks (including adverse events, QoL- and xerostomia questionnaires and laboratory testing) at the outpatient clinic. Twenty-four weeks after cycle two an end of study evaluation is planned together with another ${ }^{18} \mathrm{~F}-\mathrm{PSMA}$ PET and (whole body) MRI. Patients in the SOC arm are eligible to receive ${ }^{177}$ Lu-PSMA-I\&T after meeting the primary study objective, which is the fraction of patients who show disease progression during the study follow up. A second primary objective is the time to disease progression. Disease progression is defined as a 100\% increase in PSA from baseline or clinical progression.

(Continued on next page)

\footnotetext{
*Correspondence: james.nagarajah@radboudumc.nl

'Department of Radiology and Nuclear Medicine, Radboudumc, Geert Grooteplein Zuid 10, 6525, GA, Nijmegen, The Netherlands

Full list of author information is available at the end of the article
}

(c) The Author(s). 2020 Open Access This article is licensed under a Creative Commons Attribution 4.0 International License, which permits use, sharing, adaptation, distribution and reproduction in any medium or format, as long as you give appropriate credit to the original author(s) and the source, provide a link to the Creative Commons licence, and indicate if changes were made. The images or other third party material in this article are included in the article's Creative Commons licence, unless indicated otherwise in a credit line to the material. If material is not included in the article's Creative Commons licence and your intended use is not permitted by statutory regulation or exceeds the permitted use, you will need to obtain permission directly from the copyright holder. To view a copy of this licence, visit http://creativecommons.org/licenses/by/4.0/. The Creative Commons Public Domain Dedication waiver (http://creativecommons.org/publicdomain/zero/1.0/) applies to the data made available in this article, unless otherwise stated in a credit line to the data. 
(Continued from previous page)

Discussion: This is the first prospective randomized clinical study assessing the therapeutic efficacy and toxicity of ${ }^{177}$ LuPSMA-I\&T for patients with OHSPC.

Trial registration: Clinicaltrials.gov identifier: NCT04443062.

Keywords: Hormone sensitive prostate Cancer, Lutetium-177-PSMA, Metastases directed therapy, Oligometastases, Radioligand therapy, Urologic oncology

\section{Background}

Prostate cancer (PC) is the most common non-skin cancer in males [1]. Despite surgery or external beam radiotherapy (EBRT), approximately $20-40 \%$ of patients will eventually have a detectable prostate-specific antigen (PSA) and present with disease recurrence $[2,3]$. If there are no curative options, patients with a short PSA doubling time (e.g. < 6 months) have a poorer prognosis and early androgen deprivation therapy (ADT) is the treatment of choice [4-6]. While ADT delays disease progression of patients, it is associated with significant side effects and frequently impairs the quality of life [7]. Therefore, there is an increasing interest in treatments to postpone ADT while maintaining good quality of life.

In recent years, metastases directed therapy (MDT) (e.g. targeted surgery or EBRT) attracted much attention to postpone ADT or even with potential cure for selected patients. Particularly, patients with a limited number of metastases ( $\leq 5$ metastases), so called 'oligometastatic' PC, seem to benefit from MDT. Here, EBRT offers an ADT free survival of 14 to 29 months with solely low-grade treatment related side effects [8-12]. Therefore, several clinical trials are currently investigating $\mathrm{MDT}$ in this hormone sensitive oligometastatic setting (clinicaltrials.gov: NCT03569241, NCT04075305, NCT02170181, NCT04302454, NCT02192788, NCT02685397, NCT04115007, NCT02264379, NCT02680587, NCT03630666, NCT02274779, NCT03795207, NCT03525288, NCT03784755).

Oligometastases was first described in 1995 by Hellman and Weichselbaum [13]. This disease status became relevant when novel imaging modalities, such as prostate specific membrane antigen positron emission tomography (PSMA-PET), were introduced with better tumor detection rates compared to the conventional scans (e.g. CT or bone scans) $[14,15]$. Consequently, all the abovementioned studies utilizes PET to detect and target the tumor (metastases) [14]. The current favored PET tracers in PC are Gallium-68 $\left({ }^{68} \mathrm{Ga}\right)$ or Fluor-18 $\left({ }^{18} \mathrm{~F}\right)$ labeled prostate-specific membrane antigen (PSMA) ligands. PSMA is highly overexpressed in $>90 \%$ of PC cells and seem to increase with the aggressiveness of the tumor $[16,17]$. Several prospective studies have shown that ${ }^{68}$ Ga-PSMA PET has an excellent sensitivity and specificity (> 85 and $98 \%$, respectively) to detect PC $[15$,
18, 19]. However, PSMA ligands, such as PSMA-617 \& PSMA-I\&T, can also be labeled with beta emitters like Lutetium-177 $\left({ }^{177} \mathrm{Lu}\right)$ for radioligand therapy to deliver high local radiation doses to tumors directly [20-22].

${ }^{177} \mathrm{Lu}$ is a beta $\left(\beta^{-}\right)$radiation emitter with a maximum energy of $0.50 \mathrm{MeV}$ with maximum penetration depth of 2 $\mathrm{mm}$ and a 6.7-days half-life. ${ }^{177} \mathrm{Lu}$ labelled PSMA is a promising new therapeutic approach and frequently used in compassionate use programs worldwide [22-26]. To date, only one prospective trial of ${ }^{177}$ Lu-PSMA-617 has been published, showing efficacy in $57 \%$ of end-stage PC patients and ${ }^{177} \mathrm{Lu}-$ PSMA was generally well tolerated [20]. These observations were recently confirmed at ASCO, with the presentation of the initial results of the TheraP trial comparing ${ }^{177} \mathrm{Lu}$ PSMA-617 to cabazitaxel in mCRPC patients (NCT03392428) [26]. The pivotal trial of ${ }^{177}$ Lu-PSMA-617 in end-stage PC, called the VISION study (clinicaltrial.gov identifier: NCT03511664), is currently being finalized. However, based on the mode of action and in concordance to results from our pilot study (clinicaltrials.gov identifier: NCT03828838), ${ }^{177}$ Lu-PSMA is also highly effective in low volume disease because of high tumor uptake of PSMA targeted radioligands in small lesions, such as oHSPC [27-29]. Moreover, the favorable toxicity profile of ${ }^{177} \mathrm{Lu}-$ PSMA seen in our pilot study supports this new treatment in this setting. Hence, we initiated a prospective randomized multicenter study analyzing the efficacy of ${ }^{177} \mathrm{Lu}$-PSMA in oHSPC to postpone disease progression and averting the need for ADT.

\section{Methods and design}

The study protocol was approved by the Medical Review Ethics Committee Arnhem-Nijmegen, The Netherlands and is registered on clinicaltrials.gov (NCT04443062).

This is a two-arm randomized open label multicenter phase II study performed in Radboudumc Nijmegen, Netherlands Cancer Institute Antoni van Leeuwenhoek Hospital Amsterdam, Amsterdam University Medical Center and University Medical Center Groningen. This study will compare ${ }^{177} \mathrm{Lu}$-PSMA-I\&T MDT in oligometastatic ( $\leq 5$ metastases on ${ }^{18}$ F-PSMA PET/CT) PC to the current standard of care (SOC), which is watchful waiting till initiation of ADT [6]. This study will include 58 patients with a PSA doubling time of $<6$ months, a patient cohort prone to initiate ADT [30]. However, all 
patients, including the SOC arm, will have access to

${ }^{177} \mathrm{Lu}-\mathrm{PSMA}-\mathrm{I} \& \mathrm{~T}$ (including the follow up schedule), but only if the primary endpoint is reached and disease progression has occurred (EOT 1) and the patients are willing to undergo ${ }^{177} \mathrm{Lu}$-PSMA RLT (Fig. 1). This design enables not only a comparison of ${ }^{177} \mathrm{Lu}$-PSMA-I\&T with SOC in a randomized setting but also analyze the efficacy of ${ }^{177} \mathrm{Lu}$ PSMA-I\&T in progressing PC patients, which may violate the inclusion criteria of "oligometastatic". Moreover, with this strategy, we anticipate to prevent drop-offs in the SOC arm what the VISION study frequently encountered [31]. The therapeutic arm patients (and SOC patients after EOT 1) will receive two cycles of $7.4 \mathrm{GBq}{ }^{177} \mathrm{Lu}$-PSMAI\&T each. This is less than the current recommended schedule for end-stage PC patients with $4-6$ cycles of 7.4GBq ${ }^{177} \mathrm{Lu}-\mathrm{PSMA}$ each [20,23]. This schedule is based on the dosimetry results from our pilot study [28]. In the present study, the ligand PSMA-I\&T will be used, which was to date not yet investigated in a prospective randomized study world-wide. Nevertheless, ${ }^{177} \mathrm{Lu}$-PSMA-I\&T has shown to be efficient in retrospective setting in endstage PC patients [22].

\section{Objectives}

The primary objective is:

- To study the effect of ${ }^{177}$ Lu-PSMA-I\&T in patients with oHSPC, by comparing the fraction of patients that have disease progression and meet EOT 1 criteria within 6 months after cycle two in a group of patients that are treated with ${ }^{177} \mathrm{Lu}$-PSMA-I\&T and a group that follows the current SOC (deferred ADT).

- A second primary objective is to compare the two arms for the time to disease progression and meeting EOT 1 criteria.

\section{Secondary Objectives are:}

- To evaluate the clinical efficacy of multiple doses ${ }^{177} \mathrm{Lu}-\mathrm{PSMA}-\mathrm{I} \& \mathrm{~T}$ in patients with oHSPC by:
- The change in PSA after ${ }^{177}$ Lu-PSMA-I\&T and proportion of achieving a $\geq 50 \%$ decrease in PSA from baseline.

- The changes in uptake (SUV) of ${ }^{18}$ F-PSMA PET/ CT before and 6 months after ${ }^{177} \mathrm{Lu}$-PSMA-I\&T.

- The changes in number and size of (soft) tissue metastases on ${ }^{18}$ F-PSMA PET/CT and (whole body) MRI after ${ }^{177} \mathrm{Lu}$-PSMA-I\&T.

- To evaluate the PFS, which is defined as the time from 'cycle one, day one' to date of evidence of: clinical progression, PSA progression, or radiographic progression and death from any cause.

- Clinical progression is defined by the treating physician (e.g. increasing pain from metastases).

- PSA progression is defined as a $\geq 25 \%$ increase in PSA from nadir, with a minimum PSA of > $0,5 \mu \mathrm{g} / \mathrm{l}$ and which is confirmed by a second value $\geq 3$ weeks later (i.e. confirmed rising trend). Within the first 12 weeks after treatment administration PSA increases will be ignored in the absence of other evidence of disease progression due to possible flare phenomenon. If no decline occurs, initial date of $\geq 25 \%$ increase will be recorded [32].

- Radiographic progression is defined by the amount and size of the lesions. Where applicable Prostate Cancer Working Group 3 (PCWG3) and Response Evaluation Criteria in Solid Tumors (RECIST) v1.1 criteria will be followed

- To assess ADT free survival in patients receiving ${ }^{177} \mathrm{Lu}$-PSMA-I\&T. ADT free survival is defined by the date any ADT (e.g. bicalutamide, luteinizing hormone-releasing hormone drugs, enzalutamide, abiraterone, etc.) is started or death related to PC.

- To evaluate the tolerability and toxicity of ${ }^{177} \mathrm{Lu}$ PSMA-I\&T defined by NCI Common Terminology Criteria for Adverse Events (CTCAE) v5.0.

- To evaluate the quality of life before and up to 6 months after ${ }^{177} \mathrm{Lu}$-PSMA-I\&T using the following questionnaires: EORTC QLQ-C30, QLQ-PR25 and xerostomia inventory.

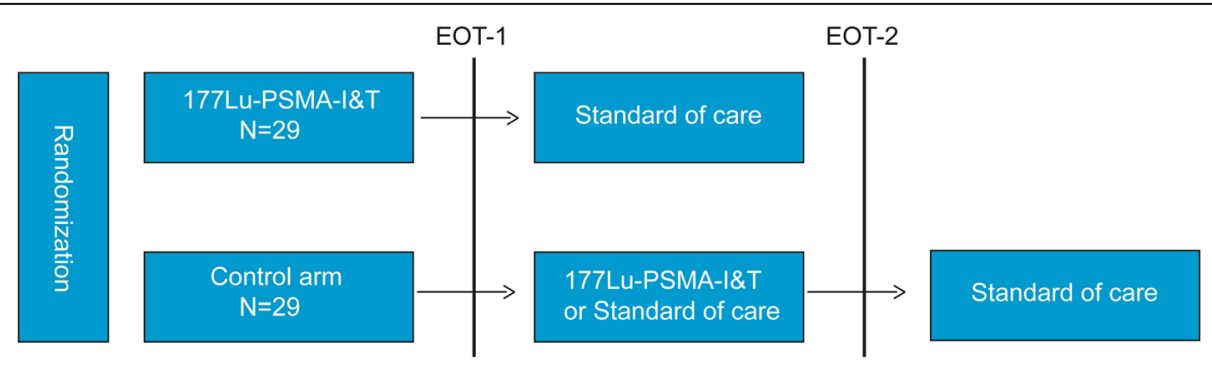

Fig. 1 A scheme of the trial with the randomization and study EOT stages. EOT=End of Treatment; PSMA = prostate specific membrane antigen 


\section{Study endpoints}

When patients have disease progression and meet EOT 1 criteria during study follow up, the primary study objective can be elucidated. EOT 1 is defined by:

- A $100 \%$ increase in PSA from 'cycle one, day one' blood draw (BASELINE) during study. Exception: PSA increase in the first 12 weeks after the first treatment injection as was defined by the PCWG3 criteria [32].

- Clinical progression determined by the treating physician (e.g. increasing pain from metastases)

After answering the primary research question (EOT $1)$, patients randomized to the SOC arm are eligible to receive ${ }^{177} \mathrm{Lu}$-PSMA-I\&T, if both the treating physician and the patient agree to continue with the trial. These study results will be analyzed separately for secondary study objectives. Although the cohorts might not be completely similar (e.g. higher tumor volume, no PSA doubling time of $<6$ months), we can include intraindividual analyses in a group that has clearly progressing PC. When these SOC patients continue to have progressive PC (defined as EOT 2) despite of ${ }^{177} \mathrm{Lu}$ PSMA-I\&T, an end of study visit should be arranged within 4 weeks, but prior to starting ADT. EOT 2 is defined by:

- A 100\% increase in PSA from 'EOT 1, cycle one, day one' blood draw (NEW BASELINE) during the study follow up. Exception: PSA increase in the first 12 weeks after the first treatment injection as was defined by the PCWG3 criteria [32].

- Clinical progression determined by the treating physician (e.g. increasing pain from metastases)

\section{Inclusion criteria}

In order to participate in this study, a subject must meet all of the following criteria:

- Histological proven adenocarcinoma of the prostate with sufficient archived tumor material. This material has to be archived till study closure.

- Biochemical recurrence (PSA > $1.0 \mu \mathrm{g} / \mathrm{l}$ ).

- PSA-doubling time $\leq 6$ months. Serum PSA progression is defined as 2 consecutive rising PSA values measured at least 1 week apart. The minimal start value is $0.2 \mu \mathrm{g} / \mathrm{l}$.

- ${ }^{18}$ F-PSMA-PET/CT positive metastases in bones and/or lymph nodes (N1/M1ab): $\geq 1$, maximally 5 metastases.

- Local treatment for oligometastases with radiotherapy or surgery appears to be no option anymore (due to prior treatment or the location of the metastatic lesions or if the patient refuses these treatments).

- No prior hormonal therapy (including any androgen directed treatment such as finasteride, dutasteride, bicalutamide, apalutamide, abiraterone or enzalutamide) or taxane based chemotherapy (docetaxel or cabazitaxel); testosterone $>1.7 \mathrm{nmol} / \mathrm{l}$.

Exception: local PC treated with local radiotherapy plus adjuvant ADT; these patients need to be stopped with ADT at least 6 months.

- A detectable lesion on the ${ }^{18}$ F-PSMA PET/CT with significant PSMA avidity, defined by a SUVmax $>15$ (partial volume corrected).

- Eastern Cooperative Oncology Group (ECOG): 0-1

- Patients must have a life expectancy $>6$ months.

- Laboratory values:

- White blood cells $>3.0 \times 10^{9} / 1$.

- Platelet count $>75 \times 10^{9} / 1$.

- Hemoglobin $>6.2 \mathrm{mmol} / \mathrm{l}$.

- Aspartate aminotransferase (AST) \& alanine aminotransferase (ALT) $<3$ x ULN.

- Glomerular filtration rate (MDRD GFR) $\geq 50 \mathrm{ml} / \mathrm{min}$

- Signed informed consent.

\section{Exclusion criteria}

A potential subject who meets any of the following criteria will be excluded from participation in this study in case of:

- A known subtype other than prostate adenocarcinoma.

- Previous PSMA based radioligand treatment.

- Visceral or brain metastases.

- Any medical condition present that in the opinion of the investigator will affect patients' clinical status when participating in this trial.

- Prior hip replacement surgery potentially influencing performance of PSMA PET/CT.

- Sjogren's syndrome

- A second active malignancy other than prostate cancer.

- Patients who are sexually active and not willing/able to use medically acceptable forms of barrier contraception.

\section{Evaluation and randomization}

All patients will have a screening visit that will include a blood draw to evaluate adequate organ functioning $(\mathrm{Hb}$, leucocytes and white blood cell differentiation, thrombocytes, creatinine, sodium, potassium, ALT, AST, LDH, alkaline phosphatase, bilirubin, gamma-glutamyl transferase, amylase, albumin, PSA and testosterone) and quality of life questionnaires (EORTC QLQ-C30, QLQ- 
PR25 and the xerostomia inventory). Furthermore, ${ }^{18} \mathrm{~F}$ PSMA PET and (whole body) MR imaging will be acquired to assess tumor PSMA uptake and heterogeneity. After reviewing all in- and exclusion criteria and study inclusion, patients will be randomized by a central reader in either the treatment or the SOC arm (1:1 ratio) using the randomization software of CastorEDC (https:// www.castoredc.com/). See Fig. 2: study flowchart.

\section{Interventions}

Once all screening or baseline procedures are performed, the next procedures will be followed within 6 weeks:

- Blood testing prior (< 7 days) to 'cycle one, day one' (for the interventional arm injection with ${ }^{177} \mathrm{Lu}$ PSMA-I\&T) for baseline assessment ( $\mathrm{Hb}$, leucocytes and white blood cell differentiation, thrombocytes, creatinine, sodium, potassium, ALT, AST, LDH, alkaline phosphatase, bilirubin, gamma-glutamyl transferase, amylase, albumin, and PSA).

- Only for ${ }^{177} \mathrm{Lu}$-PSMA-I\&T patients: the day of treatment injection ('cycle one, day one') preinfusion measurement of vital signs (respiratory rate, blood pressure and heart rate). Subsequently, an intravenous (IV) dose of PSMA-I\&T labeled with approximately $7.4 \mathrm{GBq}$ of ${ }^{177} \mathrm{Lu}$ will be slowly administered in approximately 5 min through the indwelling catheter. Following completion of the injection, a normal saline flush (approximately 10 $\mathrm{mL}$ ) will ensure that all ${ }^{177} \mathrm{Lu}$-PSMA-I\&T remaining in the infusion line is injected. The estimated radioactive dose will be determined by measuring the amount of radioactivity in the syringe pre- and postinjection, using a calibrated radioisotope dose calibrator. 30-60 min after injection vital signs (respiratory rate, heart rate and blood pressure) will be remeasured. Approximately 1-24 h after therapeutic injection, whole body imaging will be acquired using a gamma camera to exclude extravasation.

\section{Follow up}

Once 'cycle one, week one' has been completed for the SOC or ${ }^{177} \mathrm{Lu}$-PSMA-I\&T arm, the next procedures will be followed:

- One week after both treatment injections, patients that received ${ }^{177} \mathrm{Lu}$-PSMA-I\&T will be evaluated for adverse events (by phone or physical consultation). Adverse events will be scored as defined by CTCAE v5.0.

- Both the SOC and ${ }^{177}$ Lu-PSMA-I\&T arm, will be monitored at the outpatient clinic for adverse events, EOT 1 or 2 criteria and toxicity (including laboratory testing: $\mathrm{Hb}$, leucocytes and white blood cell differentiation, thrombocytes, creatinine, sodium, potassium, ALT, AST, LDH, alkaline phosphatase, bilirubin, gamma-glutamyl transferase, amylase, albumin and PSA) every third week after 'cycle one, day one' ( ${ }^{177} \mathrm{Lu}$-PSMA-I\&T application) and the week prior to the second cycle. After the second cycle, all patients will be monitored (including laboratory tests) at week $3,6,12$ \& 18 .

- To evaluate quality of life, patients will be asked to fill in the EORTC-QLQ-30, EORTC-QLQ-PR 25 and the xerostomia questionnaire at the start of each $\left({ }^{177} \mathrm{Lu}-\mathrm{PSM}-\mathrm{I} \& \mathrm{~T}\right)$ cycle and 6, 12, 18 and 24 weeks after the second therapeutic injection.

- 24 weeks after the second injection, all study patients will have an end of study visit, including laboratory testing and image acquisition of ${ }^{18} \mathrm{~F}$ PSMA PET and (whole body) MRI.

- In case of disease progression (defined as EOT 1 or 2), the end of study (whole body) MRI and ${ }^{18} \mathrm{~F}$ PSMA PET scans should be acquired within 4 weeks and prior to the start of ADT or ${ }^{177} \mathrm{Lu}$-PSMA-I\&T injection.

- SOC arm patients that have disease progression and meet EOT 1 will receive ${ }^{177} \mathrm{Lu}$-PSMA-I\&T within 6 weeks of the EOT 1 visit. These patients will follow the same procedures and follow up as the interventional am patients starting ${ }^{177} \mathrm{Lu}$-PSMA. They will receive an extra (whole body) MRI and ${ }^{18}$ F-PSMA PET at the end of the study. If disease continue to progress and someone meets EOT 2 criteria despite of ${ }^{177}$ Lu-PSMA-I\&T, an end study visit should also be planned within 4 weeks, including the extra (whole body) MRI and ${ }^{18} \mathrm{~F}$ PSMA PET scans, and prior to the start of ADT.

- After completion of the study protocol, patients will be followed according to the standard of care.

The labeling and purification of PSMA-I\&T with ${ }^{177} \mathrm{Lu}$ ${ }^{177} \mathrm{LuCl} 3$ will be obtained from ITG (Garching, Germany). Good manufacturing practice (GMP)-grade PSMA-I\&T will be obtained from piCHEM (RaabaGrambach, Austria). The radiolabeling of PSMA-I\&T will be performed on GRP synthesis module (Scintomics, Fürstenfeldbruck, Germany) using sterile and GMP-grade SC-105 kits. In brief, $4 \mathrm{mg}$ gentisic acid and the PSMA-I\&T peptide will be dissolved in $500 \mu \mathrm{L}$ WFI and added to the reaction vessel. After addition to the ${ }^{177} \mathrm{LuCl} 3$ in sodium acetate buffer and ascorbic acid the reaction will be incubated at $100{ }^{\circ} \mathrm{C}$ for $20 \mathrm{~min}$. After cooling down, the product will be diluted to $16.5 \mathrm{ml}$ with saline/DTPA to which $0.9 \mathrm{ml}$ ethanol has been added. The radioactive solution will be filtered through a $0.22 \mu \mathrm{m}$ filter (Millex GV. 


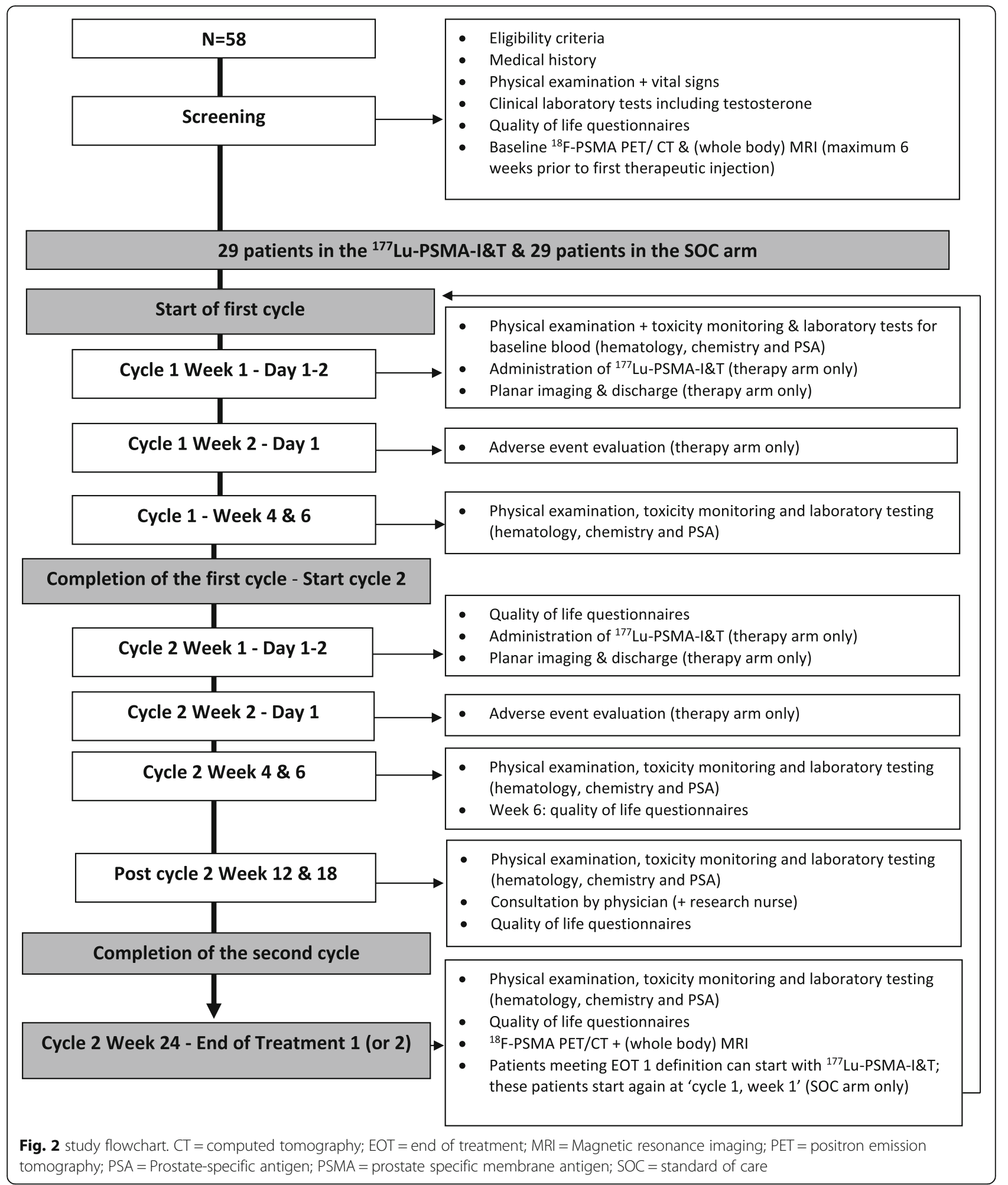

Merck, Amsterdam, The Netherlands) and dispensed into a closed glass type I container. Microbiological monitoring in class $\mathrm{C}$ will be performed during synthesis, filtration and dispensing. Assembling of the dispensing and filtration system will be performed in a class A isolator with a class B airlock (in a class C background). The radiolabeled PSMA-I\&T will be measured for total radioactivity in an appropriately calibrated radioactive dose calibrator prior to injection. 


\section{Sample size calculation}

After finishing the trial, the performance of the treatment is tested based on the first primary outcome; fraction of patients that have disease progression and meet the EOT 1 criteria within 6 months after cycle two. Only if the null hypothesis is rejected (hierarchical testing), the two arms can be compared for the second primary outcome; time till EOT 1 criteria.

Within the testing strategy as described, the sample size calculation needs to be performed for the first primary outcome only, with the risk of over- or underpowering the second-additional primary outcome. Therefore, an additional sample size calculation was performed for the second primary research question.

For the primary objective, the binomial test with pooled/equal variance under the null hypothesis and continuity correction was performed, with the numbers: sign level 0.05 , power: $80 \%$, fraction of treatment arm / control arm: $0.30 / 0.70$ at 6 months. These assumption of the fractions in the two arms were based on the pilot study and published data $[8,20,23,33-35]$. To obtain enough power for the test described above; 29 patients per arm are needed.

For the second primary objective, an exponential distribution for the time to meet EOT 1 criteria was assumed. The median survival time on the control treatment was 3.45 months (based on 0.70 at 6 months). If the true hazard ratio (relative risk) of control subjects relative to experimental subjects is 3.33 (computed based on the assumed fractions at 6 months), we will need to study 25 experimental subjects and 25 control subjects to be able to reject the null hypothesis that the experimental and control survival curves are equal with probability (power) 0.80 . The Type I error probability associated with this test of this null hypothesis is 0.05 . For a longer accrual interval (which will be the case in practice), the power will increase due to longer followup of individuals. That means that, in terms of power, the second test also has sufficient power.

\section{Data analyses}

All data is managed in CastorEDC database (https:// www.castoredc.com/). After finishing the trial, the performance of the treatment is tested based on the first primary outcome. This will be tested with a binomial test with pooled/equal variance under the null hypothesis and continuity correction. Comparison between the arms is made based on the second primary outcome, by means of the log rank test, but only if the null hypothesis of the primary objective is rejected. From both study arms sample fractions with 95\% confidence intervals and Kaplan Meier curves will be computed. The significance level is set at 0.05 .

\section{Discussion}

Currently, increasing data are showing that MDT for oHSPC improves PFS without significant side effects, in contrast to the toxicity related to ADT $[8,9,11]$. In this setting, ${ }^{177} \mathrm{Lu}$-PSMA is anticipated to be effective coupled with low grade toxicity. Moreover, ${ }^{177} \mathrm{Lu}$ PSMA-I\&T is not limited to previous curative intended treatments like surgery or EBRT. ${ }^{177} \mathrm{Lu}$-PSMA I\&T is injected intravenously and targets PSMA expressing tumors selectively. This trial will investigate if ${ }^{177} \mathrm{Lu}$ PSMA-I\&T RLT is an effective treatment in oHSPC, and is currently the first study investigating ${ }^{177} \mathrm{Lu}$-PSMAI\&T in ADT-naïve setting, but also the first randomized prospective study with PSMA-I\&T world-wide.

\section{Abbreviations \\ ${ }^{68}$ Ga: Gallium-68; ${ }^{18}$ F: Fluor-18; ${ }^{177}$ Lu: Lutetium-177; ADT: Androgen deprivation therapy; ALT: Alanine aminotransferase; AST: Aspartate aminotransferase; CT: Computed tomography; CTCAE: Common terminology criteria for adverse events; EBRT: External beam radiotherapy; ECOG: Eastern cooperative oncology group; EOT: End of study; GMP: Good manufacturing practice; IV: Intravenous; LDH: Lactate dehydrogenase; MDRD GFR: Glomerular filtration rate; MDT: Metastases directed therapy; oHSPC: Oligometastatic hormone-sensitive prostate cancer; PC: Prostate cancer; PCWG3: Prostate cancer working group 3; PET: Positron emission tomography; PSA: Prostate-specific antigen; PSMA: Prostate-specific membrane antigen; RECIST: Response evaluation criteria in solid tumors; SOC: Standard of care; SUV: Standardized uptake value}

\section{Acknowledgements}

We thank all the investigators of the study, the participating study sites, the patients and their families.

\section{Authors' contributions}

$J N, A W, W G, D O L, W V$ and $W N$ as principle investigators and $B P, M J, M G, M J$, $S M, I O, N M$ and JB as sub-investigator conceived and designed the study. BP and $\mathrm{JN}$ drafted the original study protocol. All the authors contributed to the manuscript and approved its submission. All authors are responsible for the daily running of the trial.

\section{Funding}

This study is funded by the Prostaatkankerstichting (Dutch prostate cancer foundation). The granting bodies did not have any role in the design of the study and will not have any role in collection, analysis, and interpretation of data and in writing the manuscript.

\section{Availability of data and materials}

The datasets used and/or analyzed during the current study are available from the corresponding author on reasonable request.

\section{Ethics approval and consent to participate}

The study protocol was approved by the Medical Review Ethics Committee Arnhem-Nijmegen, The Netherlands. All study participants will provide informed consent before study entry.

Consent for publication

Not applicable.

\section{Competing interests}

The authors report no conflicts of interest in this work.

\section{Author details}

'Department of Radiology and Nuclear Medicine, Radboudumc, Geert Grooteplein Zuid 10, 6525, GA, Nijmegen, The Netherlands. ${ }^{2}$ Department of Urology, Radboudumc, Nijmegen, The Netherlands. ${ }^{3}$ Department of Health Evidence, Radboudumc, Nijmegen, The Netherlands. ${ }^{4}$ Department of Medical Oncology, Radboudumc, Nijmegen, The Netherlands. ${ }^{5}$ Department of 
Radiology and Nuclear Medicine, University Medical Center Groningen, Groningen, The Netherlands. ${ }^{6}$ Department of Radiology and Nuclear Medicine, NKI Antoni van Leeuwenhoek Hospital, Amsterdam, The Netherlands. ${ }^{7}$ Department of Radiation Oncology, NKI Antoni van Leeuwenhoek Hospital, Amsterdam, The Netherlands. ${ }^{8}$ Department of Medical Oncology, NKI Antoni van Leeuwenhoek Hospital, Amsterdam, The Netherlands. 'Department of Urology, NKI Antoni van Leeuwenhoek Hospital, Amsterdam, The Netherlands. ${ }^{10}$ Department of Urology, Amsterdam University Medical Center, Amsterdam, The Netherlands. " ${ }^{1}$ Department of Radiology and Nuclear Medicine, Amsterdam University Medical Center, Amsterdam, The Netherlands.

Received: 15 August 2020 Accepted: 7 September 2020 Published online: 14 September 2020

\section{References}

1. Bray F, Ferlay J, Soerjomataram I, Siegel RL, Torre LA, Jemal A. Global cancer statistics 2018: GLOBOCAN estimates of incidence and mortality worldwide for 36 cancers in 185 countries. CA Cancer J Clin. 2018;68(6):394-424.

2. Djavan B, Moul JW, Zlotta A, Remzi M, Ravery V. PSA progression following radical prostatectomy and radiation therapy: new standards in the new millennium. Eur Urol. 2003:43(1):12-27.

3. Han M, Partin AW, Pound CR, Epstein JI, Walsh PC. Long-term biochemical disease-free and cancer-specific survival following anatomic radical retropubic prostatectomy. The 15-year Johns Hopkins experience. Urol Clin North Am. 2001;28(3):555-65.

4. Freedland SJ, Eastham J, Shore N. Androgen deprivation therapy and estrogen deficiency induced adverse effects in the treatment of prostate cancer. Prostate Cancer Prostatic Dis. 2009;12(4):333-8.

5. Zhou P, Chen MH, McLeod D, Carroll PR, Moul JW, D'Amico AV. Predictors of prostate cancer-specific mortality after radical prostatectomy or radiation therapy. J Clin Oncol. 2005;23(28):6992-8.

6. Cornford P, Bellmunt J, Bolla M, Briers E, De Santis M, Gross T, Henry AM, Joniau S, Lam TB, Mason MD, et al. EAU-ESTRO-SIOG guidelines on prostate Cancer. Part II: treatment of relapsing, metastatic, and castration-resistant prostate Cancer. Eur Urol. 2017;71(4):630-42.

7. Ahmadi H, Daneshmand S. Androgen deprivation therapy: evidence-based management of side effects. BJU Int. 2013;111(4):543-8.

8. Ost P, Reynders D, Decaestecker K, Fonteyne V, Lumen N, De Bruycker A Lambert B, Delrue L, Bultijnck R, Claeys T, et al. Surveillance or metastasisdirected therapy for Oligometastatic prostate Cancer recurrence: a prospective, randomized, multicenter phase II trial. J Clin Oncol. 2018;36(5): 446-53.

9. Fossati N, Suardi N, Gandaglia G, Bravi CA, Soligo M, Karnes RJ, Shariat S, Battaglia A, Everaerts W, Joniau S, et al. Identifying the optimal candidate for salvage lymph node dissection for nodal recurrence of prostate Cancer: results from a large, Multi-institutional Analysis. Eur Urol. 2019;75(1):176-83.

10. Aluwini SS, Mehra N, Lolkema MP, Oprea-Lager DE, Yakar D, Stoevelaar H, van der Poel H, Busstra M, de Jong IJ, de Reijke T, et al. Oligometastatic prostate Cancer: results of a Dutch multidisciplinary consensus meeting. Eur Urol Oncol. 2019;3(2):231-8.

11. Phillips R, Shi WY, Deek M, Radwan N, Lim SJ, Antonarakis ES, Rowe SP, Ross AE, Gorin MA, Deville C, et al. Outcomes of observation vs stereotactic ablative radiation for Oligometastatic prostate Cancer: the ORIOLE phase 2 randomized clinical trial. JAMA Oncol. 2020;6(5):650-9.

12. Schmidt-Hegemann NS, Kroeze SGC, Henkenberens C, Vogel MME, Kirste S, Becker J, Burger IA, Derlin T, Bartenstein P, Eiber M, et al. Influence of localization of PSMA-positive oligo-metastases on efficacy of metastasisdirected external-beam radiotherapy-a multicenter retrospective study. Eur J Nucl Med Mol Imaging. 2020;47(8):1852-63.

13. Hellman S, Weichselbaum RR. Oligometastases. J Clin Oncol. 1995;13(1):8-10.

14. Lecouvet FE, Oprea-Lager DE, Liu Y, Ost P, Bidaut L, Collette L, Deroose CM, Goffin K, Herrmann K, Hoekstra OS, et al. Use of modern imaging methods to facilitate trials of metastasis-directed therapy for oligometastatic disease in prostate cancer: a consensus recommendation from the EORTC imaging group. Lancet Oncol. 2018;19(10):e534-45.

15. Hofman MS, Lawrentschuk N, Francis RJ, Tang C, Vela I, Thomas P, Rutherford N, Martin JM, Frydenberg M, Shakher R, et al. Prostate-specific membrane antigen PET-CT in patients with high-risk prostate cancer before curative-intent surgery or radiotherapy (proPSMA): a prospective, randomised, multi-Centre study. Lancet. 2020;395(10231):1208-16.
16. Silver DA, Pellicer I, Fair WR, Heston WD, Cordon-Cardo C. Prostate-specific membrane antigen expression in normal and malignant human tissues. Clin Cancer Res. 1997;3(1):81-5.

17. Ghosh A, Heston WD. Tumor target prostate specific membrane antigen (PSMA) and its regulation in prostate cancer. J Cell Biochem. 2004;91(3): 528-39.

18. Calais J, Ceci F, Eiber M, Hope TA, Hofman MS, Rischpler C, Bach-Gansmo T, Nanni C, Savir-Baruch B, Elashoff D, et al. ${ }^{18} \mathrm{~F}$-fluciclovine PET-CT and ${ }^{68} \mathrm{Ga}-$ PSMA-11 PET-CT in patients with early biochemical recurrence after prostatectomy: a prospective, single-Centre, single-arm, comparative imaging trial. Lancet Oncol. 2019;20(9):1286-94.

19. Wondergem M, Jansen BHE, van der Zant FM, van der Sluis TM, Knol RJJ, van Kalmthout LWM, Hoekstra OS, van Moorselaar RJA, Oprea-Lager DE, Vis AN. Early lesion detection with (18)F-DCFPyL PET/CT in 248 patients with biochemically recurrent prostate cancer. Eur J Nucl Med Mol Imaging. 2019; 46(9):1911-8.

20. Hofman MS, Violet J, Hicks RJ, Ferdinandus J, Thang SP, Akhurst T, Iravani A, Kong G, Ravi Kumar A, Murphy DG, et al. [(177)Lu]-PSMA-617 radionuclide treatment in patients with metastatic castration-resistant prostate cancer (LuPSMA trial): a single-Centre, single-arm, phase 2 study. Lancet Oncol. 2018;19(6):825-33.

21. Strosberg J, El-Haddad G, Wolin E, Hendifar A, Yao J, Chasen B, Mittra E, Kunz PL, Kulke MH, Jacene H, et al. Phase 3 trial of 177Lu-Dotatate for Midgut neuroendocrine tumors. N Engl J Med. 2017;376(2):125-35.

22. Heck MM, Tauber R, Schwaiger S, Retz M, D'Alessandria C, Maurer T, Gafita A, Wester $\mathrm{H}-\mathrm{J}$, Gschwend JE, Weber WA, et al. Treatment outcome, toxicity, and predictive factors for Radioligand therapy with $<$ sup $>177</$ sup $>$ LuPSMA-I\&amp;T in metastatic castration-resistant prostate Cancer. Eur Urol. 2019;75(6):920-6.

23. Rahbar K, Ahmadzadehfar H, Kratochwil C, Haberkorn U, Schafers M, Essler M, Baum RP, Kulkarni HR, Schmidt M, Drzezga A, et al. German multicenter study investigating 177Lu-PSMA-617 Radioligand therapy in advanced prostate Cancer patients. J Nuclear Med. 2017;58(1):85-90.

24. Sathekge M, Bruchertseifer F, Knoesen O, Reyneke F, Lawal I, Lengana T, Davis C, Mahapane J, Corbett C, Vorster M, et al. 225Ac-PSMA-617 in chemotherapy-naive patients with advanced prostate cancer: a pilot study. Eur J Nucl Med Mol Imaging. 2019;46(1):129-38.

25. van Kalmthout L, Braat A, Lam M, van Leeuwaarde R, Krijger G, Ververs T, Mehra N, Bins A, Hunting J, de Keizer B. First experience with 177Lu-PSMA617 therapy for advanced prostate Cancer in the Netherlands. Clin Nucl Med. 2019;44(6):446-51.

26. Hofman MS, Emmett L, Sandhu SK, Iravani A, Joshua AM, Goh JC, Pattison $\mathrm{DA}$, Tan TH, Kirkwood ID, Ng S, et al. TheraP: A randomised phase II trial of 177Lu-PSMA-617 (LuPSMA) theranostic versus cabazitaxel in metastatic castration resistant prostate cancer (mCRPC) progressing after docetaxel: Initial results (ANZUP protocol 1603). J Clin Oncol. 2020;38(15_suppl):5500.

27. O'Donoghue JA, Bardies M, Wheldon TE. Relationships between tumor size and curability for uniformly targeted therapy with beta-emitting radionuclides. J Nuclear Med. 1995;36(10):1902-9.

28. Annual Congress of the European Association of Nuclear Medicine October 12-16, 2019 Barcelona, Spain. Eur J Nucl Med mol Imaging 2019, 46(1):1952.

29. Privé B, Peters $S$, Muselaers C, Zamecnik $P$, Janssen $M$, Scheenen $T$, Konijnenberg M, Verzijlbergen J, Gerritsen W, Mehra N et al: Lutetium-177PSMA-617 in low-volume hormone sensitive metastatic prostate cancer: a prospective study, vol. 59; 2020.

30. Gillessen S, Attard G, Beer TM, Beltran H, Bossi A, Bristow R, Carver B, Castellano D, Chung BH, Clarke N, et al. Management of Patients with advanced prostate Cancer: the report of the advanced prostate Cancer consensus conference APCCC 2017. Eur Urol. 2018;73(2):178-211.

31. Rahbar K, Bodei L, Morris MJ. Is the Vision of Radioligand therapy for prostate Cancer becoming a reality? An overview of the phase III VISION trial and its importance for the future of Theranostics. J Nucl Med. 2019; 60(11):1504-6.

32. Scher HI, Morris MJ, Stadler WM, Higano C, Basch E, Fizazi K, Antonarakis ES, Beer TM, Carducci MA, Chi KN, et al. Trial design and objectives for castration-resistant prostate Cancer: updated recommendations from the prostate Cancer clinical trials working group 3. J Clin Oncol. 2016;34(12): 1402-18.

33. Duchesne GM, Woo HH, Bassett JK, Bowe SJ, D'Este C, Frydenberg M, King M, Ledwich L, Loblaw A, Malone S, et al. Timing of androgen-deprivation 
therapy in patients with prostate cancer with a rising PSA (TROG 03.06 and VCOG PR 01-03 [TOAD]): a randomised, multicentre, non-blinded, phase 3 trial. Lancet Oncol. 2016;17(6):727-37.

34. Boorjian SA, Thompson RH, Tollefson MK, Rangel L, Bergstralh EJ, Blute ML, Karnes RJ. Long-term risk of clinical progression after biochemical recurrence following radical prostatectomy: the impact of time from surgery to recurrence. Eur Urol. 2011;59(6):893-9.

35. Studer UE, Collette L, Whelan P, Albrecht W, Casselman J, de Reijke T, Knonagel H, Loidl W, Isorna S, Sundaram SK, et al. Using PSA to guide timing of androgen deprivation in patients with TO-4 NO-2 MO prostate cancer not suitable for local curative treatment (EORTC 30891). Eur Urol. 2008;53(5):941-9.

\section{Publisher's Note}

Springer Nature remains neutral with regard to jurisdictional claims in published maps and institutional affiliations.

Ready to submit your research? Choose BMC and benefit from:

- fast, convenient online submission

- thorough peer review by experienced researchers in your field

- rapid publication on acceptance

- support for research data, including large and complex data types

- gold Open Access which fosters wider collaboration and increased citations

- maximum visibility for your research: over $100 \mathrm{M}$ website views per year

At BMC, research is always in progress.

Learn more biomedcentral.com/submissions 